\title{
Michael Haugh* and Donal Carbaugh Self-disclosure in initial interactions amongst speakers of American and Australian English
}

\begin{abstract}
Getting acquainted with others is one of the most basic interpersonal communication events. Yet there has only been a limited number of studies that have examined variation in the interactional practices through which unacquainted persons become acquainted and establish relationships across speakers of the same language. The current study focuses on self-disclosure practices in initial interactions between first language speakers of English from Australia and the United States. It was found that while both American and Australian participants volunteered self-disclosures in the context of presentation-eliciting questions, there was a noticeable tendency for the American participants to selfdisclose without being prompted by questions from the other participant. We also found that there was a tendency for the Australians to use positive assessments in response to self-disclosures less often and with a lesser degree of intensity than the American participants. These tendencies in self-disclosure practices are argued to reflect the ways in which underlying cultural premises are used by participants. However, given that a significant degree of interspeaker and same-speaker variability was also observed, it is concluded that the study of pragmatic variation be situated on the level of interactional routines, relational dyads, and upwards that are engaged in particular social activities.
\end{abstract}

Keywords: self-presentation, superlatives, Australian English, American English, getting acquainted

DOI 10.1515/multi-2014-0104

\footnotetext{
*Corresponding author: Michael Haugh, School of Languages and Linguistics, Griffith University, Brisbane, QLD 4111, Australia, E-mail: m.haugh@griffith.edu.au Donal Carbaugh, Department of Communication, University of Massachusetts, Amherst, MA, USA, E-mail: carbaugh@comm.umass.edu
} 


\section{Introduction}

Getting acquainted with others is one of the most basic interpersonal communication events. Yet much of the research on the interactional practices through which persons become acquainted and establish relationships - whether they be passing or ongoing - has been undertaken from a social psychological perspective, where the data have been primarily gleaned from questionnaires, and the focus has been primarily on the importance of uncertainty reduction or increasing relational trust through self-disclosure. There have more recently, however, been a growing number of studies that are grounded in the actual details of talk in initial face-to-face interactions across different languages such as English (e.g. Carbaugh 2005; Haugh 2011; Maynard and Zimmermann 1984; Pillet-Shore 2011; Schneider 1988; Silverstein 1984), Swedish (Svennevig 1999, Svennevig 2014), and Japanese (Usami 2002). Such studies have explored the ways in which self-presentation is sequentially accomplished and its role in forming relationships (Maynard and Zimmermann 1984; Pillet-Shore 2011; Svennevig 2014; Schneider 2008), the topics that arise in such interactions (Schneider 1987), the role of humour (Haugh 2010, Haugh 2011), and interpersonal dimensions of interaction more generally (Schneider 1988; Svennevig 2014; Usami 2002).

One aspect that has received less attention, however, is the extent to which there may be variation in the pragmatics of initial interactions, particularly amongst speakers of languages such as English for which there are a large number of recognised varieties distributed throughout the world (Barron and Schneider 2005; Schneider and Barron 2008; Schneider 2010). With just a few exceptions (Carbaugh 2005; Schneider 2008, Schneider 2012; see also Goddard 2012a, Goddard 2012b), there has been little research on initial interactions from the perspective of variation in such practices across speakers of the same language. Yet while limited in number, these studies have been highly suggestive in raising the distinct possibility that there may well be variation in practices and forms that arise in initial interactions, and in how these might be interpreted across speakers of different varieties of English.

In the current study, we focus on initial interactions where first language speakers of English from Australia and the United States are getting acquainted and highlight salient variation in practices relating to self-disclosure. Drawing from analytical approaches in interactional pragmatics (Arundale 2010a; Haugh 2012, 2014, 2015) and cultural discourse analysis (Berry 2009; Carbaugh 2007; Scollo 2011) we analyse interactions in intracultural settings where Australians and Americans are getting acquainted with other Australians and Americans, 
respectively. In the course of this analysis we identify a number of putative similarities and differences in the sequential organisation of self-disclosure practices, as well as the cultural interpretative value placed on these different practices from an emic or cultural insider's perspective.

It is important to note, however, that we are not advocating a cultural essentialist model where speakers of different varieties of English can be expected to behave or even think in particular ways. Such a viewpoint has been the subject of ongoing critique more generally in cross-cultural research (e.g. Berry 2009; Fuchs 2001; Holliday 1999), and is also inconsistent with the stated importance of various kinds of different macro-factors in variational pragmatics (Schneider 2010). However, it is nevertheless our contention that there may well be a greater emphasis on particular practices due to the cultural interpretative value that can be recognisably and accountably placed on them by those speakers. In other words, while we explicitly argue that one cannot claim that, say Americans or Australians, will behave in predictable ways in initial interactions given that these interactions are invariably locally situated and torqued, we propose that there are nonetheless interesting similarities and differences that can be observed through close analysis of such initial interactions. We further propose that these practices may be found to be consonant with broader cultural premises that have been observed as immanent in social interactional practices amongst speakers of those varieties of English. While generalising to all members of a culture from studies of interaction amongst a select number of members is clearly problematic, this is not to say that culture does not play an important role in such interactions. Our view is thus that participants in initial interactions may accountably orient to their respective national identities through various means in the course of those interactions. Consequently, these orientations to (national) identities can play a role in moulding and influencing both the ways in which those sequences develop, as well as the interpretations of practices and (symbolic) forms for which those participants may be held accountable in the course of such interactions.

We begin this paper by first briefly reviewing prior research on initial interactions. We start by outlining work on sequential aspects of getting acquainted before moving on to consider prior research on interpersonal and then cross-cultural aspects of initial interactions. After describing the dataset that informs our subsequent claims about self-disclosure practices amongst American and Australian speakers of English, and the methodological framework that underpins that analysis, we move to our analysis of these self-disclosure practices proper. Building on prior work on self-presentation in initial interactions (Stokoe 2010; Svennevig 1999, Svennevig 2014), we develop an 
account of the sequential organisation of self-disclosure, focusing, in particular, on a distinction we draw between prompted and unprompted self-disclosures. We next examine the relative occurrence of two different types of unprompted self-disclosures in a selected, matched sample of interactions drawn from the larger dataset that enables us to explore possible inter-group as well as interspeaker and intra-speaker variation in these practices. We then move to consider the relative occurrence of superlatives and positive assessments in response to self-disclosures across the two matched sub-samples, thereby once again allowing us to explore these different levels of pragmatic variation. Our analysis of unprompted self-disclosures and evaluative responses to self-disclosure across American and Australian speakers of English is also considered in the course of this analysis in light of the cultural premises that these participants appear to be using in getting acquainted. We conclude by reflecting on the implications of our study for the field of variational pragmatics more generally.

\section{Getting acquainted in face-to-face interaction}

Previous studies have identified a number of key areas of interest in relation to the pragmatics of getting acquainted. A number of studies have converged on the importance of the sequential organisation of initial interactions, including the ways in which greetings and introductions are accomplished (Pillet-Shore 2010, Pillet-Shore 2011, and Pillet-Shore 2012) and how these may differ across varieties of English (Schneider 2008, Schneider 2012). For instance, based on the results of discourse production tasks, Schneider (2008) suggests that American speakers of English display a preference for disclosing their identity following a greeting (e.g. Hi, I'm Ashley), while Irish speakers of English display a preference for some kind of positive evaluation of the event that has brought about the initial contact following a greeting (e.g. Hi! Great party, isn't it?). This, in turn, contrasts with English speakers of English who display a preference for bare greetings (e.g. $\mathrm{Hi}$ ). The way in which self-presentation, or more specifically, selfdisclosure in the form of talk about biographical/personal information or community membership to an unknowing recipient is accomplished in initial interactions has also been the subject of analysis (Stokoe 2010; Maynard and Zimmermann 1984; Svennevig 1999 and Svennevig 2014; Usami 2002). Svennevig $(1999,2014)$, for instance, claims that initial interactions are characterised as instances of getting acquainted through the occurrence of what he terms the "self-presentation sequence", where a presentation-eliciting question occasions a self-disclosure on the part of the recipient, which the prior speaker 
either topicalises to elicit further self-disclosure by the recipient, or volunteers personal information through a self-directed comment. ${ }^{1}$

Another area of interest has centred on common topics in initial interactions (Usami 2002), or what Schneider $(1987,1988)$ terms “small talk". These include topics relating to the immediate situation, the broader external situation, and the communication situation. However, it is generally only the latter that involve self-disclosure on the part of participants. Schneider (1987) suggests that the "starting point for small talk is always the medium level" (254), namely, topics relating to the "larger context of the immediate situation" (254), which include things such as the connection of the interlocutors to that situation (e.g. who do you know at this party).

Interpersonal aspects of initial interactions have also been studied. Schneider (1988), for instance, proposes an analytical framework that attempts to tease out the "politeness maxims" underpinning "small talk" - or events of getting acquainted - in initial interactions, including the need to avoid lapses of talk and the need to show interest in the other, along with the preference for indicating agreement and positive evaluations. The potentially important role that humour plays in getting acquainted in interactions amongst Australian speakers of English (Haugh 2011) and Japanese (Usami 2002) has also been the subject of research. In addition, a number of studies of interpersonal aspects of initial interactions have focused on Brown and Levinson's (1987) politeness strategies (e.g. Svennevig 1999, Svennevig 2014; Usami 2002). In such studies it is proposed that getting acquainted involves attempts by participants to find "mutually involving topics" in order to establish "some degree of familiarity, solidarity and affection" (Svennevig 2014: 322). One difficulty that couching the analysis of interpersonal dimensions of getting acquainted in terms of Brown and Levinson's (1987) theory of politeness creates, however, is that there is little discussion of what counts as "showing approval" (i.e. orienting to "positive face"), and whether the tokens of this type vary across different languages and cultures. More problematically it leaves unanswered the question of whether "politeness strategies" in getting acquainted are just a matter of avoiding face threats as Brown and Levinson (1987) would appear to have it, or whether there is deeper cultural work getting done in such interactions.

1 It is worth noting in passing that this research has been undertaken without much reference to the large body of work in North American Communication on self-disclosure more generally (e.g. Derlega et al. 1993; Dindia 2000; Pearce and Sharp 1973; Petronio 2002). This current paper is intended to be complementary to that previous body of work in communication in offering fine-grained analyses of the interactional mechanics of self-disclosures in initial interactions. 
Such questions lead us to a consideration of studies that have touched upon cross-cultural aspects of getting acquainted. Cross-cultural studies have generally been dominated by broad generalisations about aspects of communication practice amongst a particular group of speakers. For example, it has been claimed that Americans compliment a lot (Tottie 2002; Goddard 2012a, Goddard 2012b), talk about themselves a lot, and do not value self-deprecation (Tottie 2002). In contrast, it has been claimed that Anglo-Australians tend to downplay achievement and favour self-deprecation (Goddard 2006, Goddard 2012a, and Goddard 2012b) as part of the broader qualitative or cultural value said to be placed on not taking oneself too seriously (Goddard 2009). However, while studies such as these hint at cultural variability in the conduct of talk itself, they often proceed with a view of talk which overlooks specific uses of languages in context as well as the cultural features in that very talk. We argue that what is needed is a more richly nuanced view of the cultural features of talk itself, in addition to an interpretation of the cultural premises which underpin its recognisability and moral values. We see and hear these cultural features and premises as both immanent in social interactional practices and as revealed through close analyses of actual interactional data (Carbaugh 2005, Carbaugh 2012). A long tradition of such work exists, but it seems to have been missed by some who study conversation.

\section{Data and method}

\subsection{Dataset}

The dataset that informs the analysis in this study is part of a larger project investigating ways in which American and Australian speakers get acquainted in face-to-face interaction. ${ }^{2}$ The overall dataset currently consists of 46 dyadic interactions between Americans and Australians that were recorded in Brisbane from August 2012 through to July 2014. The participants were invited to take part in the project, which was broadly construed as one about communication in English, as a way of getting to know new people. In each case, two participants were taken into a room after which it was explained to them that they were being recorded for the purposes of a study of communication. No

2 The authors wish to acknowledge the support of a Discovery grant from the Australian Research Council (DP120100516) that has enabled the research reported in this paper to be undertaken. 
further instruction about what they should talk about was given. Instead they were invited to talk about whatever they wished. In most cases the participants were invited to stop the recording after approximately twenty minutes in order to enable them to get back to work or attend classes. However, the actual recordings themselves vary in length from 15 and a half minutes through to 1 hour and 50 minutes, with the mean length being close to 26 minutes. The participants were independently motivated to meet new people as participation was entirely voluntary and did not involve any financial inducement. That getting to know people was indeed the aim of many participants was also evidenced by the fact that a number of these encounters resulted in further contact being made between those participants on their own initiative.

In total, the interactions amount to 1,171 minutes of audiovisual recordings (approximately 19 and a half hours), or an estimated 250,000 transcribed tokens of getting acquainted episodes. Of these interactions, 23 were in male-female pairs, 15 were in female-female pairs, and 8 were in male-male pairs. 62 participants were involved in these 46 recordings as some of them participated in more than one interaction. 36 of the participants were female and 26 were male, hence the relative imbalance in female-female versus male-male interactions. The participants ranged in age from 17 through to 61, but most were in their early twenties through to mid-thirties (17 24 years old $=26 ; 25 \sim 35$ years $=25 ; 36+$ years $=11$ ). Perhaps as a consequence of the recordings having been made in Australia, the American participants were not representative of different ethnic groups in the U.S., but were primarily white, non-Hispanics, although they came from a variety of regions across the continental U.S., including those regions broadly described as North-Eastern, Southern, MidWestern and Western United States. They had all been in Australia less than two years. The Australian participants were also not representative of ethnic diversity in Australia, but were all white Australians, and although they came from a variety of regions around Australia, they were predominantly from Queensland. All participants were broadly middle class, professionals or students that were university educated according to self-reports in the participant background questionnaire.

The participants in the dataset are thus not considered to be representative of the two national cultures in question, given the considerable diversity in both cases. Indeed, in light of the detailed interactional analysis required in our study, we elected to select a matched sample of ten interactions involving five Australian-Australian and five American-American getting acquainted episodes for detailed annotation. The sub-sample of five Australian-Australian initial interactions was matched with respect to gender-mix and age with a sub-sample of five American-American initial interactions, each of which consisted of 
approximately 20,000 tokens (i.e. including not only "words", but also discourse particles and response tokens). The interactions were selected in order to allow not only an intergroup comparison to be undertaken, but also to check for possible intra-group (or inter-speaker) variability as well as intra-speaker variability, in recognition of the potential importance of inter-individual variation that lies below the level of aggregated categories (Brezina and Meyerhoff 2014: 21), in this case, American and Australian speakers of English. These ten interactions were then independently annotated in relation to the frequency of self-disclosures that occurred in response to a question from the other participants (what we term "prompted self-disclosures), self-disclosures that occurred outside the context of a question from the other participant (what we term "unprompted self-disclosures"), as well as the number of positive evaluative responses to self-disclosures. ${ }^{3}$ Agreement about cases where there were initial inconsistencies (approximately 10\% of instances of unprompted self-disclosures and positive evaluative responses) was reached through subsequent discussion between the two annotators. ${ }^{4}$

\subsection{Analytical framework and approach}

Given our focus on exploring pragmatic variation in initial interactions, the current study was by necessity informed both by corpus-assisted methods in interactional pragmatics and cultural discourse analysis.

Interactional pragmatics is an approach to pragmatic phenomena that is informed by research and methods in ethnomethodological conversation analysis (Arundale 2010a; Haugh 2012, Haugh 2015). As outlined in Culpeper and Haugh (2014), pragmatics can be broadly understood as the study of language in use, but more specifically as "the study, by observer-analysts, of what particular form-function relationships are taken to mean by user-participants in particular

3 What constitutes a hearable absence of a positive evaluative response was found to be difficult to consistently annotate across the interactions given that continuers were often used in response to (un)prompted self-disclosures. It was thus (analytically) equivocal in a number of cases whether a positive evaluative response was properly due at that point. However, further investigation of the absence of an expected response to self-disclosures, if indeed positive evaluative responses are properly due in some sequential environments, is something, we would suggest, that deserves further consideration in future work.

4 While a sub-sample of ten interactions may appear, at first glance at least, to be relatively small, the annotation process required very close sequential analysis, which arguably confers some degree of validity on the analysis. Employing a second annotator also arguably confers some degree of reliability on the analysis. The first author would like to thank Lara Weinglass, who is the research assistant on this project, for her assistance in annotating this sub-sample. 
situated, sequential contexts, and how this can vary across those participants" (266). These interactional meanings encompass pragmatic meanings (i.e. what participants are taken to be referring to, presuming, saying, implying, inferring and so on), pragmatic acts (i.e. what kinds of socially recognisable acts and activities those participants are taken to be doing), and the interpersonal relations, attitudes and evaluations they are taken to be instantiating (Culpeper and Haugh 2014: 267). The emphasis in interactional pragmatics, or integrative pragmatics more broadly, then, is on interactional meanings and both the awareness of participants about those meanings (i.e. metapragmatic awareness) and variation in the situated instantiation of those meanings. Notably, while the emphasis in interactional pragmatics is on detailed analyses of pragmatic phenomenon in the situated, sequential contexts in which they occur, it is not assumed that such an approach requires eschewing more quantitatively oriented corpus-based methods (Haugh 2014).

Cultural discourse analysis is a philosophy of communication based in ethnographic studies of actual social practices (Berry 2009; Carbaugh 2007; Scollo 2011). Its theory - by focusing on participants' practices which code social action itself, identities, social relations, emotion, and place - draws attention to the cultural foundations of conversation practices (Carbaugh 2005, Carbaugh 2007). Its methodology follows several modes which involve the distinct and complementary analytic paths of descriptive, interpretive, comparative and critical study. From this view, culture is conceptualised as both a condition for and an activity within conversation (Carbaugh 2012: 74), where cultural discourse refers to a "historically transmitted expressive system of communication practices, of acts, events, and styles, which are composed of specific symbols, symbolic forms, norms and their meanings" (Carbaugh 2007: 169). In this way, the approach offers a communication theory of culture as active in social praxis generally and in conversation specifically.

Broadly speaking, the descriptive dimension of this study was carried out within an interactional pragmatics framework, while the cultural interpretative aspect of the study was carried out within a cultural discourse analytic framework. However, during the analysis we found these two approaches were mutually informing in various ways, and so a hard and fast division of labour was neither intended nor practised in the course of our analysis.

The analysis of self-disclosure practices began in our study with repeated viewings of recordings of getting acquainted episodes taken from the larger dataset. While a number of practices in relation to greetings and introductions, topic selection and development, the role of teasing and jocular self-deprecation, and the impact of all of these on the development of the relationship between those participants, emerged as salient themes, a number of similarities as well as 
differences in the set of practices by which participants accomplished self-disclosure were also noticed in the course of this broad initial analysis of the dataset. Self-disclosures themselves were operationally defined as talk about biographical/ personal information or community membership to an unknowing recipient (Stokoe 2010; Svennevig 2014). Our definition thus includes what Derlega et al. (1993) term “descriptive self-disclosure”, namely, information about oneself that is more or less personal, and "relational self-disclosure", namely, information about one's relationship with others. It excludes, however, "evaluative self-disclosure”, which involves the expression of personal feelings, opinions or judgements, as the latter arguably make contingently relevant different responses (e.g. agreeing or disagreeing responses) than the former two (e.g. acknowledgements or positive assessments).

We then undertook a close, qualitative analysis of sequences where selfdisclosures arose, with the aim being to better understand the sequential environments in which self-disclosures occur, their sequential organisation, as well as the responses of participants to such self-disclosures. Two key noticings emerged through repeated viewings of such interactional sequences, namely, that selfdisclosures tended to occur less often in the context of a preceding question in the case of American participants, and that Australian participants tended to offer less positive evaluations in response to self-disclosures than American participants. These initial qualitative analyses then formed the basis of the comparative analysis of self-disclosures practices in initial interactions between American and Australian speakers of English that is reported in the following section.

\section{Self-disclosure practices across two varieties of English}

In this section, we first report on our overall findings about the sequential organisation of self-disclosure in initial interaction that emerged from our analysis of the whole dataset. We then move, in the following sub-sections, to discuss the results of our annotation of the frequency of prompted self-disclosures, unprompted self-disclosures, and positive evaluative responses to selfdisclosures in the matched sub-sample of initial interactions.

\subsection{The sequential organisation of self-disclosure}

There were numerous instances where self-disclosures were prompted through recognisable questions on the part of the prior speaker. These self-disclosures 
thus occurred in the context of the self-presentation sequences described in general terms by Svennevig (1999, 2014). In some cases these self-disclosures were formulated in such a way as to only address the terms of the question, thereby constituting a minimal response. In other cases, in contrast, the selfdiscloser volunteered information that addressed the broader (semantic) agenda of the question, thereby constituting a non-minimal or extended response. In such responses, the question-recipient volunteers more than is strictly speaking being asked for, although the response nevertheless generally addresses the putative agenda of that prior speaker's question. ${ }^{5}$ These kinds of minimal and non-minimal self-disclosures, both prompted by questions by the prior speaker, were prevalent in the talk of both American and Australian participants. In addition to these prompted self-disclosures we also observed a number of instances of self-disclosures that were unprompted, namely self-disclosures which did not arise in the vicinity of either a direct or indirect question (cf. Stokoe 2010: 264). Such unprompted self-disclosures were found to be either sequential-initial, that is, launching a new social action or stance sequence (Stivers 2013) distinct from that which came prior to it, or sequence-medial, that is, not launching a new social action or stance sequence, but rather positioned as responsive to a prior (un)prompted self-disclosure. ${ }^{6}$ These different types of self-disclosure practices are summarised in Figure 1 below.

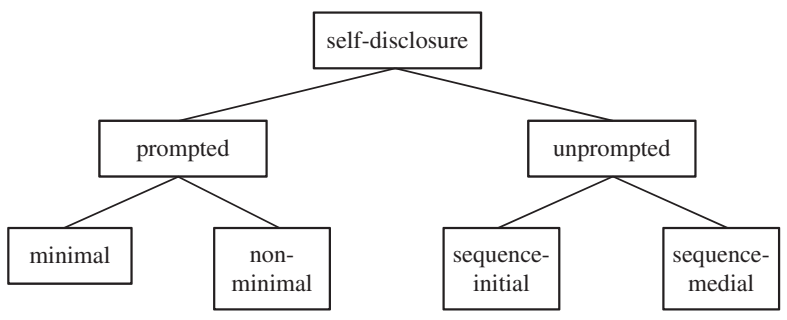

Figure 1: Sequential organisation of self-disclosures.

5 Prompted self-disclosures were sequence-initial by definition given that they followed presentation-eliciting questions. We did not count self-disclosures that followed continuer questions within our count of prompted self-disclosures as this shaded into an analysis of topicalisation.

6 All the unprompted self-disclosures we found in our dataset were "non-minimal contributions", in the sense that they all consisted of complex 'sentences', and there were no instances of unprompted self-disclosures that consisted of single words or phrases (as was the case in some instances of non-minimal responses to presentation-eliciting questions). 
The minimal and non-minimal prompted self-disclosures evident in excerpt (1) below are illustrative of the kinds of prompted self-disclosures we identified across the larger dataset. ${ }^{7}$

(1) AusAus04: 00:39

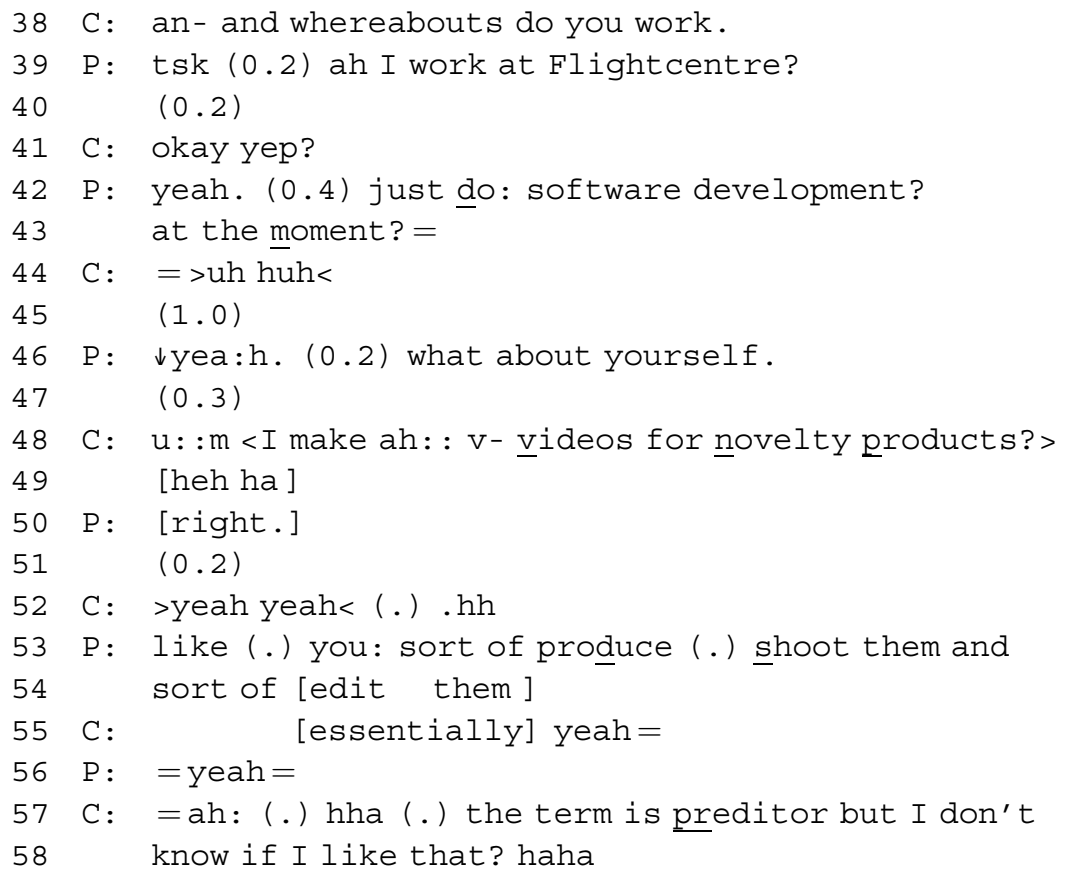

The first self-presentation sequence in the above excerpt begins with a presentation-eliciting question by Chris (line 38), which constitutes a non-minimal response as Peter not only orients to the terms of the question in indicating where he works (line 39), he also volunteers further information about the type of work he does (lines 42-43) following an initial acknowledgement by Chris (line 41). Peter then reciprocates the same presentation-eliciting question (line 46), which occasions a minimal response from Chris that treats Peter's question as being one about what kind of work Chris does (line 48). A continuation elicitor by Peter (lines 53-54) then offers a candidate understanding of what kind of work is involved (Pomerantz 1988), which elicits both an affirmative response (line 55), and then further elaboration about this type of work (lines 57

7 The excerpts in this paper are all transcribed using standard CA transcription conventions (Jefferson 2004; Hepburn and Bolden 2013). 
onwards). The two participants thereby accomplish a mutual orientation to Chris's work as the topic in question through this second self-presentation sequence (Svennevig 2014).

Unprompted self-disclosures, in contrast, emerged outside the scope of a presentation-eliciting question. In some cases, these unprompted self-disclosures worked to initiate a new topical sequence, as seen in excerpt (2) below, or what are here termed sequence-initiating unprompted self-disclosures.

(2) AmAm03: 4:06

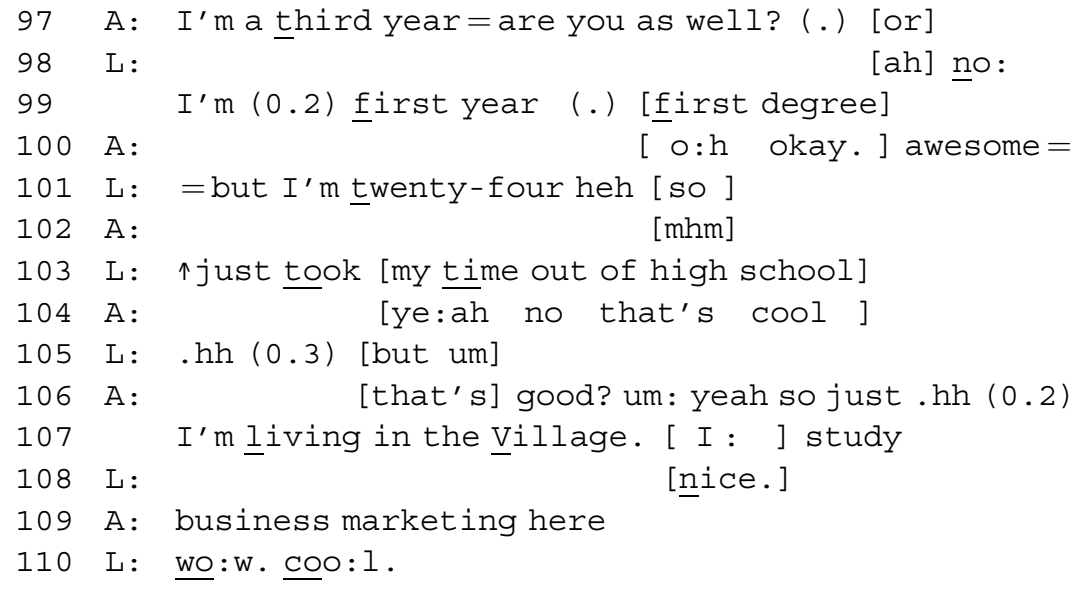

The above excerpt begins with a presentation-eliciting question from Ann about what stage Liz is at in relation to her progression of study (line 97), thereby occasioning a non-minimal self-disclosure by Liz (lines 98-105) as part of a fairly typical self-presentation sequence. However, in line 106, subsequent to the expression of an affiliative stance in response to Liz's extended self-disclosure, Ann launches a self-disclosure about where she lives and what she studies that has not been prompted by any explicit or implicit question on the part of Liz. In other words, Ann launches a distinct sequence focusing on where she lives and what she studies through a stepwise topic transition (Jefferson 1984). It is in this sense, then, that it constitutes a sequence-initiating unprompted selfdisclosure.

Unprompted self-disclosures were also found to occur in other sequencemedial positions. In excerpt (3), we can observe examples of sequence-medial unprompted self-disclosures that arise as part of an account for the speaker's stance, namely, his view that the results of this "research" about communication will be interesting. 
(3) AusAus09: 0:44

42 T: so I'd be really interested to see ho: w. (.)

43 [ all ] of this pans out $i: n(0.3)$ in the face

$44 \mathrm{~N}: \quad\left[{ }^{\circ} \mathrm{yep}^{\circ}\right]$

$45 \mathrm{~T}:$ of [their] research? =

$46 \mathrm{~N}: \quad[$.hhh ]

47 T: [and] things like that?

$48 \mathrm{~N}:$ [yep]

$49 \mathrm{~N}:{ }^{\circ}$ yep. ${ }^{\circ}$

$50 \mathrm{~T}:$.hh it's interesting I did um: (.) I'm at- (0.2)

51 I did- $(0.3)$ uh a teaching degree.

$52 \mathrm{~N}:$ yeah? =

$53 \mathrm{~T}$ : = um but did some language? study as part of that?

$54 \mathrm{~N}:$ yep?

$55 \mathrm{~T}$ : and was a language teacher? for a little while?

56 [as well] and .hh (0.2) anything relating to: (0.2)

$57 \mathrm{~N}:$ [ yeah ]

$58 \mathrm{~T}$ : um: (.) language an- linguistics and stuff I find

59 rea:lly $\cdot$ hh

$60 \mathrm{~N}:$ yeah $=$

$61 \mathrm{~T}:$ = interesting? =

$62 \mathrm{~N}:=\underline{\mathrm{ye}}: \mathrm{s} .=$

$63 \mathrm{~T}:=\mathrm{sO}=$

$64 \mathrm{~N}:=[$ same $]$

$65 \mathrm{~T}:=\left[\right.$ that' $\left.^{\mathrm{s}}\right]$ why $[\mathrm{I}-$

$66 \mathrm{~N}$ : [an-an-also just manners and

67 etiquette

$68 \mathrm{~N}$ : did you travel a lot with your job?

After taking a positive stance on the research project (lines 42-47), Tim then goes on to offer an account for this stance, namely, that he has studied linguistics and was also a language teacher, and this is why he's interested in research about communication (lines 50-65). Embedded within this account are a number of selfdisclosures, namely, that he has a teaching degree and that he's been a language teacher. These unprompted self-disclosures occasion affiliative responses from Nicky with respect to Tim's stance on the project (lines 62, 64, 66-67), and subsequently a presentation-eliciting question about where Tim has worked (line 68).

Overall, then, through close analysis of the dataset it was found that selfdisclosures could be broadly divided into those that are prompted or unprompted. In examining the occurrence of self-disclosure across the dataset, however, it became evident that while all of these practices are indeed enacted by both American and Australian participants, their relative frequency of 
occurrence was not consistent across the two groups, and, further, that responses to these self-disclosures were not qualitatively of the same type. In particular, it was observed that while there were a similar number of prompted self-disclosures amongst American and Australian participants, American participants tended to offer unprompted self-disclosures more often, and that Australian participants tended to offer positive evaluations in response to selfdisclosures less frequently. However, this is not to say, as we have noted, that the latter practices were not readily available to Australians. The question facing us, then, was whether these observed tendencies represent potentially robust cross-cultural differences in self-disclosure practices in initial interactions. It is to this question that we now turn to consider in the following three sub-sections.

\subsection{Prompted self-disclosures}

Prompted self-disclosures involve one participant making reference to biographical information (i.e. descriptive self-disclosure) or membership with respect to some group or other party (i.e. relational self-disclosure) to another unknowing recipient in response to a sequentially contiguous presentation-eliciting question on the part of that other participant. In the matched sub-sample of five American-American and five Australian-Australian initial interactions a count of sequence-initial and sequence-medial unprompted self-disclosures yielded a total of 70 instances in the American-American sample (25,531 tokens) and 72 in the Australian-Australian sample (19,138 tokens). These raw frequencies were then normalised with respect to the number of tokens produced by each speaker in their respective conversations, as illustrated in Table 1 below.

Table 1: Raw and normalised frequency of prompted self-disclosures in American-American and Australian-Australian initial interactions.

\begin{tabular}{llrr}
\hline & & $\begin{array}{r}\text { American- } \\
\text { American }\end{array}$ & $\begin{array}{r}\text { Australian- } \\
\text { Australian }\end{array}$ \\
\hline Minimal & Raw frequency & 25 & 19 \\
& Normalised frequency $(/ 1,000$ tokens $)$ & 0.98 & 0.99 \\
\multirow{2}{*}{ Non-minimal } & 45 & 53 \\
& Raw frequency & 1.76 & 2.77 \\
\cline { 2 - 3 } Total & Normalised frequency (/1,000 tokens) & 70 & 72 \\
& Raw frequency & 2.74 & 3.76 \\
\hline
\end{tabular}


While the Likelihood-ratio test (Rayson 2008) is often used to test whether differences in frequency of occurrence between samples of talk from corpora are statistically significant, we elected to use a Mann-Whitney U test (Brezina and Meyerhoff 2014) in order to counter the effect of any inter-speaker variation in the use of unprompted self-disclosures in initial interactions in both the American and Australian samples. ${ }^{8}$ This indicated that despite the greater relative frequency of non-minimal prompted self-disclosures by Australian participants, the difference was not statistically significant on a two-tailed, non-directional test $\left(\mathrm{U}_{\mathrm{A}}=69\right.$; $p=0.1615)$. The difference in the relative frequency of minimal and non-minimal prompted self-disclosures was also not statistically significant (UA=61; $p=0.4295$ ), which was not surprising given there was minimal difference in the relative frequency of minimal prompted self-disclosures. In other words, there was no statistically significant difference in the relative frequency of prompted selfdisclosures between American and Australian participants. It follows, then, that American and Australian participants were proffering presentation-eliciting questions in similar numbers in these initial interactions.

\subsection{Unprompted self-disclosures}

Unprompted self-disclosures involve one participant making reference to biographical information or membership with respect to some group to another unknowing recipient without the disclosure having been occasioned by any sequentially contiguous question on the part of that other participant. In the matched subsample of five American-American and five Australian-Australian initial interactions a count of sequence-initial and sequence-medial unprompted self-disclosures yielded a total of 153 instances in the American-American sample (25,531 tokens) and 67 in the Australian-Australian sample (19,138 tokens). These raw frequencies were then normalised with respect to the number of tokens produced by each speaker in their respective conversations, as illustrated in Table 2 below.

An analysis using the Mann-Whitney $U$ test indicated that while the difference in the total number of unprompted self-disclosures in the annotated sample of initial interactions between Americans versus Australians was indeed statistically significant on a two-tailed, non-directional test $\left(\mathrm{U}_{\mathrm{A}}=89 ; p=0.0036\right)$, as was the difference in the number of sequence-medial unprompted self-disclosures $\left(\mathrm{U}_{\mathrm{A}}=88, p=0.0047\right)$, there was not a statistically significant difference in regards to the relative frequency of sequence-initial unprompted self-disclosures $\left(\mathrm{U}_{\mathrm{A}}=75, p=0.0643\right)$. In other words, it was the relatively higher frequency of

8 These were calculated using the online calculator at http://vassarstats.net. 
Table 2: Raw and normalised frequency of unprompted self-disclosures in American-American and Australian-Australian initial interactions.

\begin{tabular}{llrr}
\hline & & $\begin{array}{r}\text { American- } \\
\text { American }\end{array}$ & $\begin{array}{r}\text { Australian- } \\
\text { Australian }\end{array}$ \\
\hline Sequence-initial & Raw frequency & 55 & 30 \\
& Normalised frequency (/1,000 tokens) & 2.15 & 1.57 \\
Sequence-medial & 98 & 37 \\
& Raw frequency & 3.84 & 1.93 \\
& Normalised frequency (/1,000 tokens) & 153 & 67 \\
& Raw frequency & 5.99 & 3.50 \\
\hline
\end{tabular}

sequence-medial unprompted self-disclosures in initial interactions between Americans that appeared to be a statistically more robust trend in this sample.

Excerpt (4) is illustrative of the kinds of sequence-medial, unprompted selfdisclosures that were frequently embedded by American participants in sequences organised around topics or stances. All self-disclosures in this excerpt are unprompted and sequence-medial. Prior to the point where this excerpt begins, John and Elizabeth have been talking about their experiences on roller coasters in theme parks in the U.S.

(4) AmAm02: 8:48

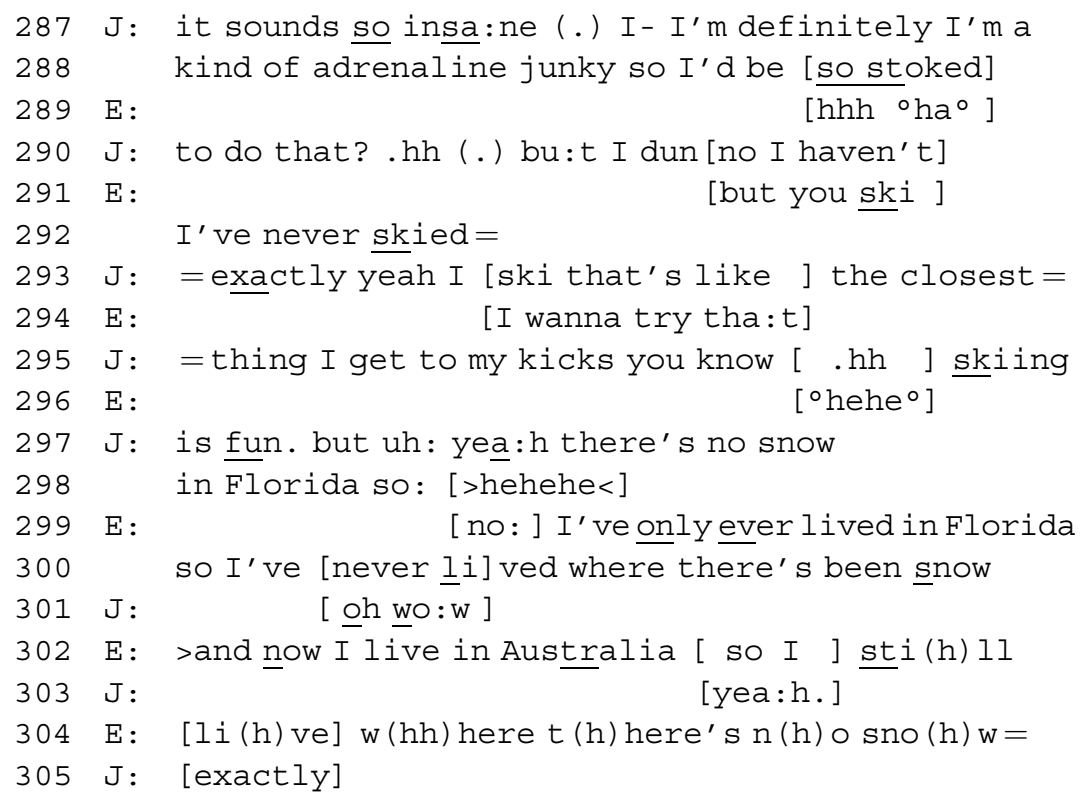




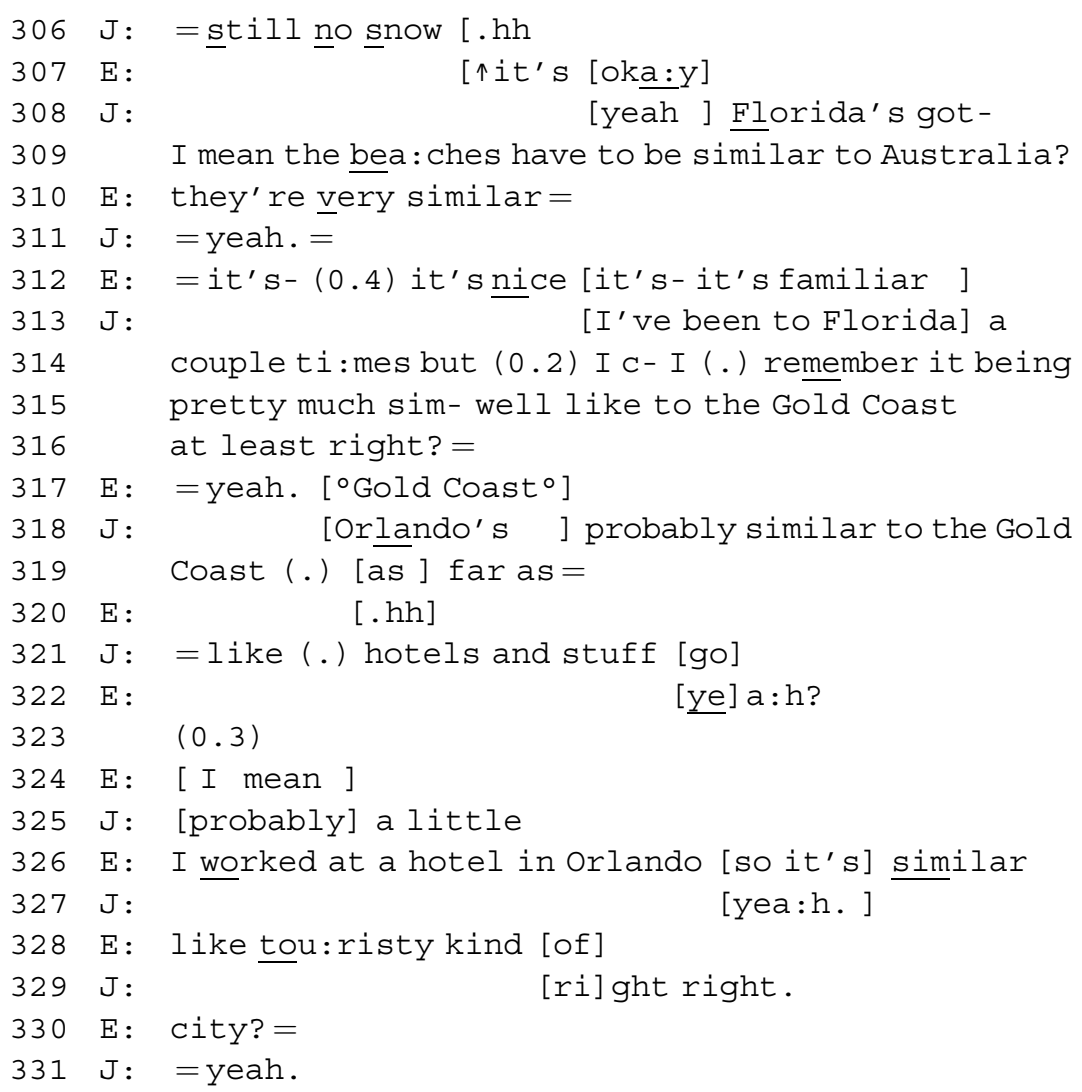

John's stance in line 287 that "it sounds so insane" is in reference to the Kingda Ka roller coaster at the Six Flags Great Adventure theme park in Jackson, New Jersey, that he'd like to try which accounts for his subsequent embedded unprompted self-disclosure that he is an "adrenaline junky" (line 288). A stepwise topic transition (Jefferson 1984) is then accomplished by Elizabeth whose embedded unprompted self-disclosure about having not been skiing before (line 292) proffers a new topic-sequence, namely, skiing. Notably, this topic proffer (Button and Casey 1984, 1985) is both recipient-oriented in that it makes reference to a prior unprompted self-disclosure by John that he is a "big skier" (data not shown), ${ }^{9}$ and thereby the inference that his skiing is consistent with his claim to be an adrenaline junky (line 288), as well as self-oriented in that it

9 The sequence initiated by Elizabeth's reference to John's prior unprompted self-disclosure thus constitutes a candidate "retro sequence" (Schegloff 2007), although space does not permit further exploration of this possibility in this paper. 
makes reference to Elizabeth's lack of experience in that respect, as well as her desire to try (line 294). This then occasions agreement from John with Elizabeth's inference that links these two prior unprompted self-disclosures (lines 293, 295), as well as expressions of affiliative encouragement from John that "skiing is fun" (lines 295, 297), before he goes on to offer a putative account for why Elizabeth hasn't skied before, namely, the lack of snow in Florida (lines 297-298). While the seriousness of this putative account is treated as ambivalent by John through turn-final laughter (Glenn 2003; Holt 2013; Jefferson 1979), which constitutes a possible invitation to Elizabeth to join this laughter about the absurdity of the idea of attempting to ski in Florida, Elizabeth simply agrees with John's proposition without any immediate acceptance of this invitation to laugh. She then offers another embedded unprompted self-disclosure, namely, that she hasn't lived anywhere else in the U.S. apart from Florida (lines 299-300), thereby accounting for the fact that she has not skied before. She then subsequently indexes that her stated desire to try skiing is problematic through interpolated laughter particles (Potter and Hepburn 2010) in the course of making reference to the fact that she now lives in (a part of) Australia where there is also no opportunity to ski (lines 302, 304). After affiliating with Elizabeth's ironic stance about the lack of opportunities for her to try skiing (lines 303, 305), John accomplishes a stepwise topic transition to talking about "beaches" in Australia versus Florida (lines 308-309). Another embedded unprompted selfdisclosure by John then arises when he makes reference to having been to Florida "a couple of times" (lines 313-314) in the course of expressing his stance that the Gold Coast and Florida are fairly similar (lines 314-321). Notably, embedded within Elizabeth's subsequent agreement with John's stance (lines 322-330) is another unprompted self-disclosure, namely, that she's worked in a hotel at Orlando (line 326).

In sum, then, in the course of talking about skiing, beaches and comparing the Gold Coast with Florida both John and Elizabeth embed a number of unprompted self-disclosures in the course of expressing their stances on those topics. In doing so they repeatedly "personalise" those stances with respect to their own background experiences (Carbaugh 2002). As argued by Carbaugh (2005: 45),

Americans believe that one should express one's self, with very few constraints being placed upon that expressiveness. In fact, participants often state the rule that people are free to say whatever they please, and it is not up to those present - thinking perhaps it is only up to God - to evaluate or judge those expressions [ . . . ] Such speaking often elaborates one's personal experiences, thoughts, and feelings. Americans are often, in public speech, then, less preoccupied with the social worthiness of their expression, than they are with its personal importance. 
Our contention is that unprompted self-disclosures constitute a practice by which this emphasis on personalising and expressing one's self is accomplished in initial interactions.

Our analysis of the matched samples of initial interactions thus appears suggestive of a tendency for the American participants to self-disclose more frequently without prompting through questions from the other participant, particularly in the course of joint topical talk or narrative tellings by the other participant. This tendency appears statistically significant despite some degree of inter-speaker variation evident upon examination of these two samples. However, while taking into account inter-individual variation that lies below the level of aggregated categories is critical when testing the statistical significance of apparent trends across samples of talk taken from corpora, as argued by Brezina and Meyerhoff (2014), we found through closer analysis of our data that we also need to take intraspeaker variation more seriously in examining different kinds of pragmatic variation. In examining the frequency of sequence-initiating, unprompted self-disclosures in initial interactions amongst the Australian participants, for instance, we found that there was as much difference in the use of unprompted self-disclosures by the same speaker across different interactions (i.e. intra-speaker variation), as there was by different speakers in the same interaction (i.e. inter-speaker variation). For instance, while one Australian participant used on average 4.02 sequentialinitial unprompted self-disclosures/1,000 tokens in one interaction, no sequentialinitial unprompted self-disclosures were used in another interaction featuring that very same speaker (hence an intra-speaker range of 4.02/1,000 tokens). In contrast, the greatest degree of difference in relation to the use of sequential-initial unprompted self-disclosures by different speakers was found to be from 1.62 through to 4.02/1,000 tokens, hence an inter-speaker range of 2.4 self-disclosures/ 1,000 tokens. We also found a very similar range in same-speaker variation (a difference of up to 3.5 self-disclosures/1,000 tokens) compared to the range of inter-speaker variation (a difference of up to 3.6 self-disclosures/1,000 tokens) in examining the frequency of sequence-medial, unprompted self-disclosures in initial interactions amongst Australian participants. A similar trend was observable in the initial interactions amongst American participants, where there was little difference in the amount of inter-speaker variation versus same-speaker variation in the case of sequence-initial unprompted self-disclosures (3.3 and 2.5 self-disclosures/1,000 tokens, respectively), although the difference was greater in the case of sequencemedial unprompted self-disclosures (4.0 and 1.5 self-disclosures/1,000 tokens, respectively).

What this suggests is that we should not be treating the talk of speakers as independent of the participant(s) with whom they are engaged in interaction. How often and in what ways one participant self-discloses can have an influence 
on how often and in what ways the other participant self-discloses. It is in this sense, then, that while observable trends in inter-group pragmatic variation may indeed represent an orientation to different cultural premises on the part of those participants, pragmatic features also invariably arise relative to locally situated, sequential contexts. Yet it also suggests that the particular array of selfdisclosure practices that are interactionally achieved in a particular interaction may also involve mutual reinforcing of putative cultural premises. In other words, one participant orienting to the cultural value placed on "personalising" through embedding self-disclosures in topical talk or the narrative tellings of others may, in turn, occasion a greater orientation to "personalising" in this way on the part of the other participant.

This tendency towards a mirroring of self-disclosure practices on the part of participants reflects, perhaps, the general value placed on reciprocity amongst both Australian and American participants in these initial interactions. This orientation to reciprocity means one participant self-disclosing in a particular way more frequently may engender the other participant to self-disclose in the same way and to do so more frequently. This was a trend that was noticeable in examining the frequency of self-disclosures across the ten interactions we annotated. The value placed on reciprocity can cut both ways, however, in that if one participant self-discloses frequently - whether prompted or unprompted - without the same degree of self-disclosure being practised on the part of the other participant, this may be open to sanction as constituting a moral transgression on the part of the one self-disclosing more often.

In the excerpt (5) below, for instance, which is taken from an initial interaction between two Australians, we can observe just such an orientation to the value placed on reciprocity in relation to self-disclosure. ${ }^{10}$

(5) AGA: ERCH: 13:31

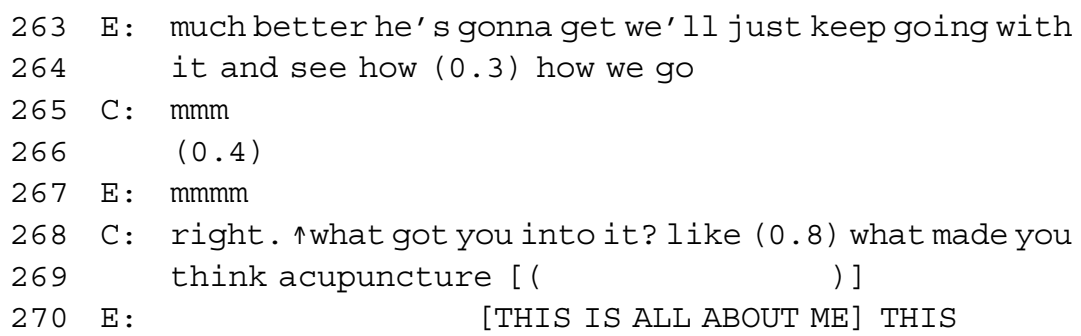

10 This excerpt is taken from the Australians Getting Acquainted (AGA) corpus, which consists of 18 audio-recorded conversations between Australians totalling around 200 minutes that was collected in Brisbane in January-February 2002. 


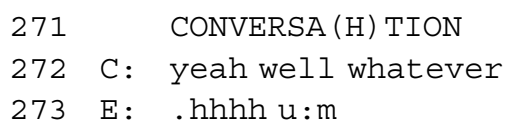

Following her response to a prior question from Chris (lines 263-264), Chris formulates another presentation-eliciting question (lines 268-269). However, Emma interrupts this question with a noticing delivered at a markedly louder volume that she has been talking too much about herself, thus implying that this is somehow problematic.

Ultimately, then, it is important to bear in mind that any putative intergroup pragmatic variation in the frequency of unprompted self-disclosures should not be analysed without taking into account inter-speaker, and indeed same-speaker pragmatic variation, a point which we will be further exploring in the following section, where we move to discuss evaluative responses to selfdisclosures in these initial interactions.

\subsection{Positive assessments and self-disclosure}

Responses to self-disclosures can involve indicators of recognition or understanding (e.g. right, okay), new understanding (e.g. oh), continuers (e.g. yeah), and so on. In some cases, however, participants may respond to self-disclosures with positive evaluations, in the form of superlative-like expressions (e.g. awesome, brilliant, cool, fantastic, perfect, great, sweet) or hearably less "exaggerated" positive assessments (e.g. lovely, good, nice). ${ }^{11}$ It has been noted by some that superlative-like expressions occur relatively frequently in talk amongst American speakers of English (Carbaugh 1997, Carbaugh 2005; cf. Goddard 2012b: 112). An initial analysis of the relative frequency of these superlative-like expressions in the matched samples of initial interactions amongst American and Australian speakers of English seemed to bear out this claim, as illustrated in Table 3 below.

Ignoring inter-speaker variation for the moment, this broad inter-group difference was found to be statistically significant based on a preliminary Likelihood-ratio test (Rayson 2008) at the 1\% level (log likelihood: 10.80;

11 The superlative-like expression "wow" is primarily an indicator of "being impressed", which can in some contexts imply a positive evaluation. However, given that this is not always the case, it was not included in the list of positive assessments for annotation. 
Table 3: Raw and normalised frequency of superlative-like expressions in initial interactions.

\begin{tabular}{lrr}
\hline & AusAus & AmAm \\
\hline Raw frequency & 92 & 173 \\
Normalised frequency (/1,000 tokens) & 4.81 & 7.29 \\
\hline
\end{tabular}

significant at $p<0.01) .{ }^{12}$ Notably, while 21 instances of these superlative-like expressions were accompanied by intensifiers in the initial interactions amongst Americans, just one was preceded by an intensifier in the interactions amongst Australian participants.

Closer analysis of the superlative-like positive assessments indicated that a much smaller portion of them were found in response to self-disclosures, as illustrated in Table 4 below.

Table 4: Raw and normalised frequency of superlative-like expressions in response to self-disclosures in initial interactions.

\begin{tabular}{lrr}
\hline & AusAus & AmAm \\
\hline Raw frequency & 23 & 49 \\
Normalised frequency (/1,000 tokens) & 1.20 & 2.06 \\
\hline
\end{tabular}

Once again a Likelihood-ratio test focusing on inter-group difference indicated this difference was significant, although only just at the $5 \%$ level (log likelihood: 4.84; significant at $p<0.05$ ). These two findings are suggestive of two distinct possibilities. One, the American participants were not necessarily using superlative-like expressions that much more frequently than Australian participants in response to self-disclosures. Second, that these superlative-like expressions were also doing other things in these initial interactions, such as accomplishing positive responses to disclosures about third parties or positive descriptions within tellings about self, third parties or events.

We thus moved to examine positive assessments in responses to selfdisclosures to see whether a more robust trend might emerge. As summarised in Table 5, there was a slightly stronger tendency for positive assessments overall that is, including both superlative-like and less emphatic ones - to occur in response to self-disclosures amongst American as opposed to Australian participants in initial interactions.

12 This was calculated using the online calculator at http://ucrel.lancs.ac.uk/llwizard.html. 
Table 5: Raw and normalised frequency of positive assessments in response to self-disclosures in initial interactions.

\begin{tabular}{lrr}
\hline & AusAus & AmAm \\
\hline Raw frequency & 36 & 90 \\
Normalised frequency (/1,000 tokens) & 1.20 & 2.40 \\
\hline
\end{tabular}

While an initial Likelihood-ratio test focusing on inter-group variation indicated that this difference is statistically significant at the $0.1 \%$ level (log likelihood: 13.75; significant at $p<0.001$ ), we elected to also conduct a Mann Whitney-U test given that some degree of inter-speaker variability emerged in the course of our more detailed annotation of positive assessments in response to self-disclosures. This yielded a less convincing trend, thus lending further credence to Brezina and Meyerhoff's (2014) cautioning researchers about the way in which aggregated data can yield what appears to be a highly significant difference that may be partly a function of inter-speaker variability rather than inter-group variability. While on a directional test, the higher frequency of positive assessments was found to be statistically significant at the $5 \%$ level $\left(\mathrm{U}_{\mathrm{A}}=74, p=0.0375\right)$, on a non-directional test it was found not to be significant $(p=0.0751)$. If one takes the view, given existing claims in the literature, that Americans use superlatives and thus presumably positive assessments more than other speakers of English (e.g. Goddard 2012a, Goddard 2012b), then the use of a directional test is perhaps justified..$^{13}$ However, we would suggest that this result remains equivocal at best.

This somewhat equivocal result, statistically speaking, reflects the high degree of inter-speaker variability in the use of positive assessments in response to selfdisclosures amongst both American (ranging from 0 to 9.8 positive assessments/ 1,000 tokens) and Australian (ranging from 0 to 8.0 positive assessments/1,000 tokens) participants in initial interactions. There was also, however, a fair degree of same-speaker variability in the use of positive assessments in this sequential context, with the greatest range being $3.8 / 1,000$ tokens (i.e. 0.6 to 4.4 positive assessments per 1,000 tokens) in the case of one of the Australian participants,

13 A directional test presumes there is a systematic effect in place driving any differences between sample groups. In this case, the assumption that Americans are likely to use positive assessments in response to self-disclosures because Americans are said by other researchers (e.g. Goddard 2012a, Goddard 2012b) to use positive assessments more frequently than speakers of other varieties of English, including Australian speakers of English. A non-directional test does not assume that such a systematic influence is in place. In other words, American participants are not assumed to be more likely to use positive assessments, and Australian participants are not assumed to be less likely to use them. 
and 6.9/1,000 tokens (i.e. 2.9 to 9.8 positive assessments per 1,000 tokens) in the case of one of the American participants in the matched sample. This suggests, once again, that the frequency of positive assessments in response to self-disclosures is, in part, a function of locally situated, sequential contexts, in that how often and in what ways one participant offers positive assessments in response to self-disclosures can have an influence on how often and in what ways the other participant offers such positive assessments. In other words, we should not be analysing pragmatic variation with respect to individual speakers in isolation, but rather as part of emergent relational systems (Arundale 2010b; Haugh 2012) where the basic unit of analysis is social interactional practices (Carbaugh 2005). One consequence of our emphasis on strips of interactional practice as a key unit of analysis is that any generalisations we make are about interaction itself, predicated on strips of practical routines, not about individual participants. Such an approach contrasts, we contend, with traditional approaches to variation where the behaviour or report of individuals is aggregated and then claimed to be representative of groups or populations of speakers.

However, putting aside the issue of whether or not American dyads tend to use positive assessments in response to self-disclosures more frequently than Australian dyads in initial interactions, it was clear from our analysis of where these positive assessments occurred in the sequences that there was a distinct tendency for these American dyads to use positive assessments more intensely over a relatively brief span of talk. We have already seen an example of this kind of clustering of positive assessments in excerpt (2) above (lines 100, 104, 110). A clustering of positive assessments can also be seen in excerpt (6) where John and Liz talk about part-time jobs.

(6) AmAm01: 14:10

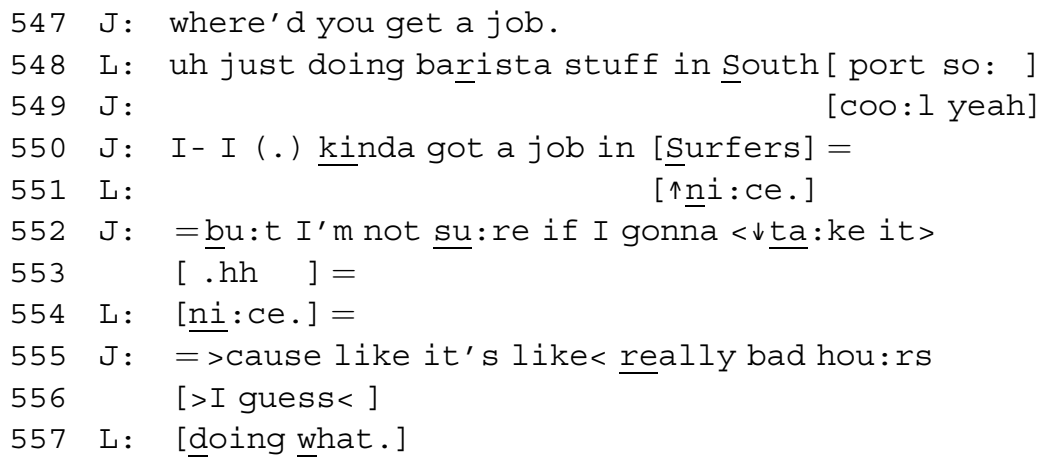

What is noticeable here is not only that Liz reciprocates (in lines 551 and 554) John's use of a positive assessment in response to a self-disclosure (in line 549), 
the second positive assessment is delivered subsequent to a self-disclosure where something else other than an assessment seems to have been made conditionally relevant, such as soliciting an account or some recognition of the newsworthiness of John's musings here. In that respect, the latter functions more like a continuer in some respects than a vehicle for accomplishing a positive assessing as such. However, no matter whether these expressions are taken to be a vehicle for accomplishing positive assessments or as merely continuers, it is evident that their use on Liz's part seems to be "done in the spirit of friendliness, of getting along with each other, even if this doesn't appear, from the outside, to go very deep" (Carbaugh 2005: 46).

Finally, while we found that there was a noticeable clustering of positive assessments in response to self-disclosures that occurred in initial interactions amongst the American dyads, this did not occur in the case of Australian getting acquainted episodes bar one exception, which is given in (7).

(7) AusAus03: 3:18

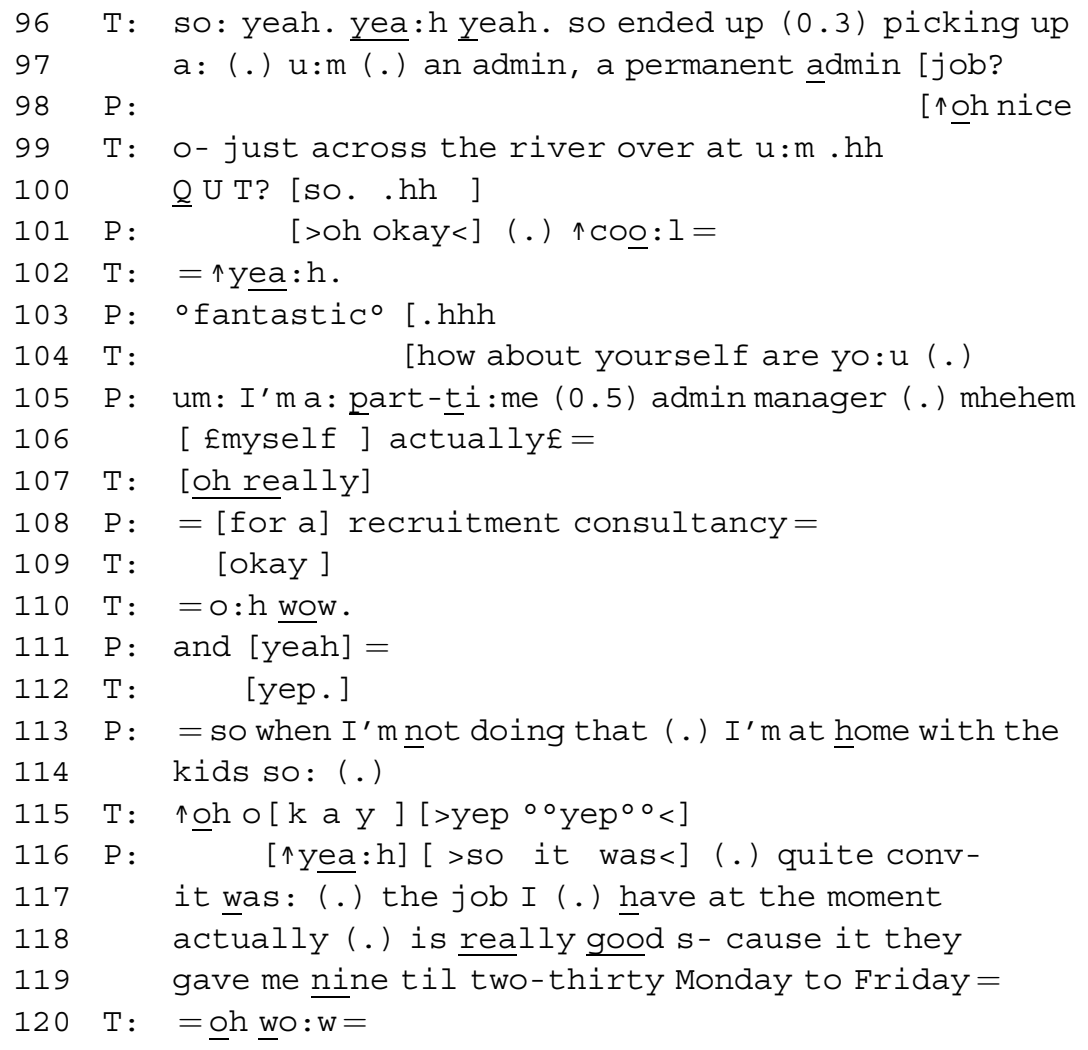




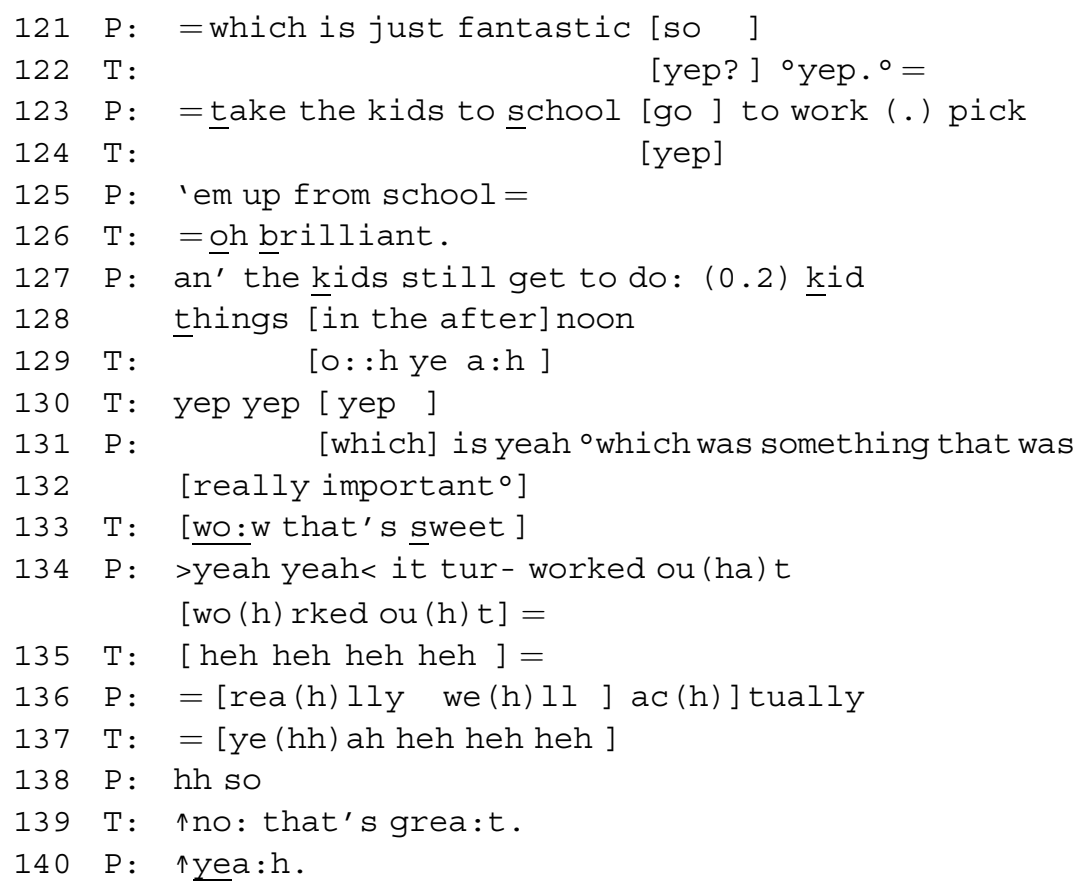

Here Pam delivers three positive assessments in three consecutive turns in response to self-disclosures by Tim (lines 98, 101, 103). Tim does not initially use positive assessments but rather "wow" in response to a series of selfdisclosures by Pam that are occasioned by a presentation-eliciting question (line 104), and, notably, these occur across sequentially non-contiguous turns (lines 110, 120). It is only subsequent to Pam using a positive assessment in relation to her own self-disclosure ("fantastic" in line 121), thereby indicating a positive stance about her current work arrangements vis-à-vis family responsibilities, that Tim starts to deliver a number of positive assessments (lines 126, 133, 139). These positive assessments thus allow Tim to affiliate, that is, to agree, with Pam's previously expressed stance. It is also noticeable that the way in which things have "worked out" for Pam is treated as a laughable by both herself and Tim (lines 134-137), indicating that Tim's positive assessment of Pam's current work arrangements may have been hearable as overly earnest (cf. Haugh 2014; Holt 2013). This line of interpretation is further supported by Tim's subsequent no-prefaced positive assessment (cf. Schegloff 2001) in line 139, which positions his subsequent positive assessment as serious vis-à-vis Pam's prior self-disclosure, and thus indicating serious (i.e. sincere) agreement with her positive stance with respect to that. The relatively frequent use of positive 
assessments in response to self-disclosures seems to have occurred, at least in the case of Tim, in the context of affiliating with the prior speaker's stance.

In sum, then, we found a marked tendency for positive assessments in response to self-disclosures to occur more frequently and with greater intensity (i.e. in clusters across only a few turns of talk) in initial interactions amongst Americans compared to Australian dyads. This arguably reflects the greater emphasis on showing "reciprocal approval" that has been observed in ethnographic studies of USAmericans (Arundale 2006; Stewart and Bennett 1991; cf. Brown and Levinson 1987), and the emphasis on "acknowledging each other's presence and linking with each other" (Carbaugh 2005: 46). Amongst Australians, on the other hand, the basic need for approval from others appeared to be tempered by a cultural premise valuing "social solidarity and social equality" (Goddard 2006, Goddard 2012a, and Goddard 2012b). However, we would not go so far as to say that the Australians explicitly avoided seeking recognition of their achievements in these initial interactions (cf. Goddard 2012a: 1041). Indeed, the Australian participants were also able to articulate a "clear sense of self" through their self-disclosures (cf. Goddard 2012a, Goddard 2012b: 110), despite this being something which Goddard (2012a, 2012b) associates primarily with Anglo-Americans. The key differences, then, seemed to lie in the way in which these self-disclosures were accomplished less frequently as unprompted amongst the Australian participants, and the way in which positive assessments in response to self-disclosures did not occur in rapid-fire clusters with frequent use of intensifiers to the same degree amongst Australian participants as was found to be the case more prevalently amongst the American participants in initial interactions.

\section{Implications}

In our analysis of initial interactions amongst a select sample of white, nonHispanic Americans and white Australians we found that a range of self-disclosure practices were available and practised by speakers from both groups. In both American and Australian dyads, participants volunteered non-minimal selfdisclosures in the context of presentation-eliciting questions. However, there was a noticeable tendency for the American participants to self-disclose more frequently in sequential contexts where they were unprompted by any question from the other participant. We also found that there was a tendency for the Australians to use positive assessments in response to self-disclosures less often and with less frequent use of intensifiers than the American participants. These tendencies in self-disclosure practices are arguably important in the way in which 
they can be interpreted with respect to the presentation of self. Prompted selfdisclosures, on the one hand, arguably amount to a type of other-influenced sharing of self (i.e. I share what I think you want me to share). Unprompted selfdisclosures, on the other hand, amount to a type of agentive or relatively autonomous sharing of self (i.e. I share what I want to share).

Such tendencies thus not only reflect subtle nuances in the underlying cultural premises about self-presentation in initial interactions that may be oriented to by American and Australian participants, but perhaps more critically represent an area where there is a danger of "invisible misunderstandings", where the frequent use of unprompted self-disclosures by an American may not be recognised as reflecting an orientation to an underlying cultural premise, namely, the value placed on "personalising" amongst USAmericans (Carbaugh 2002, Carbaugh 2005). In this way, then, we have highlighted the importance of studies in variational pragmatics more broadly (Barron and Schneider 2005; Schneider 2010, Schneider 2012; Schneider and Barron 2008) for the analysis of interactions amongst speakers of polycentric languages such as English.

However, we would nevertheless strongly caution against any generalisations being extended to Americans and Australians more broadly from this study, even in the restricted sense of so-called Anglo-Americans and AngloAustralians. This is in part because the sample of initial interactions is not large enough to reliably make generalisations about such a large group of speakers. However, it is also in part because alongside this apparent inter-group pragmatic variation, we have also observed a significant degree of inter-speaker and same-speaker variability. It is now commonly acknowledged that individual speakers are not so-called Parsonian cultural dopes who act out internalised cultural norms, as has been long argued (e.g. Garfinkel 1967). Thus, we would suggest that not only should studies of inter-group variation take such interspeaker variability properly into account, but we also need to appropriately acknowledge the degree to which the same speaker may vary in their situationally located practices when interacting with different interlocutors. The importance of acknowledging the likely existence of same-speaker variability for studies in variational pragmatics is that rather than researchers necessarily assuming that variability is always located in individuals, that in many cases such variability emerges through relational systems (Arundale 2006, Arundale 2010b; Haugh 2012). In other words, we should be studying pragmatic variation at the level of relational dyads and upwards to larger relational groupings, alongside studies of pragmatic variation amongst aggregates of individuals. We can then further interpret what cultural premises are active in the practices that arise in the context of such dyadic, or larger, multi-party interactions (cf. Carbaugh et al. 2012). 
Other questions also remain unanswered by the current study - including the role of other macro-social variables such as region, gender, age, ethnicity and so on - some of which can be explored in this dataset (e.g. gender and age), and some of which require further data (e.g. ethnicity). But no matter what the focus of subsequent work in variational pragmatics, we would suggest that what are required are quantitative analyses that are grounded in close, interactional analyses, which thereby recognise not only putative macro-social variation, but also inter-speaker as well as intra-speaker variation (see Berry 2009). The latter also points strongly to the need to recognise that all interactions are invariably shaped by the locally situated contingencies of those participants in question. Indeed, our analysis suggests that any study of macro-social factors in isolation will inevitably bleach out the ways in which participants are inevitably engaged not only in inter-group but also inter-personal interaction. It is thus incumbent on studies in variational pragmatics to work towards a theory of pragmatic variation that accounts for both the simultaneously intergroup and interpersonal dimensions of that variation.

\section{References}

Arundale, Robert. 2006. Face as relational and interactional: A communication framework for research on face, facework, and politeness. Journal of Politeness Research 2(2). 193-216.

Arundale, Robert. 2010a. Constituting face in conversation: Face, facework and interactional achievement. Journal of Pragmatics 42(8). 2078-2105.

Arundale, Robert. 2010b. Relating. In Miriam Locher \& Sage Lambert Graham (eds.), Interpersonal pragmatics, 137-166. Berlin: Mouton de Gruyter.

Barron, Anne \& Klaus P. Schneider (eds.). 2005. The pragmatics of Irish English. Berlin: Mouton de Gruyter.

Berry, Michael. 2009. The social and cultural realization of diversity: An interview with Donal Carbaugh. Language and Intercultural Communication 9. 230-241.

Brezina, Vaclav \& Miriam Meyerhoff. 2014. Significant or random? A critical review of sociolinguistic generalisations based on large corpora. International Journal of Corpus Linguistics 19(1). 1-28.

Brown, Penelope \& Stephen Levinson. 1987. Politeness. Cambridge: Cambridge University Press. Button, Graham \& Neil Casey. 1984. Generating topic. In J. Maxwell Atkinson \& John C. Heritage (eds.), Structures of social action, 167-190. Cambridge: Cambridge University Press.

Button, Graham \& Neil Casey. 1985. Topic nomination and topic pursuit. Human Studies 8(1). 3-55.

Carbaugh, Donal. 1997. Finnish and American linguistic patterns: A cultural comparison. In Larry A. Samovar \& Richard E. Porter (eds.), Intercultural communication: A reader. 8th edn., 221-226. Belmont, CA: Wadsworth Publishing Company.

Carbaugh, Donal. 2002. Some distinctive features of U.S. American conversation. In William F. Eadie \& Paul E. Nelson (eds.), The changing conversation in America, 61-75. Thousand Oaks, CA: Sage.

Carbaugh, Donal. 2005. Cultures in conversation. London: Routledge. 
Carbaugh, Donal. 2007. Cultural discourse analysis: Communication practices and intercultural encounters. Journal of Intercultural Communication Research 36(3). 167-182.

Carbaugh, Donal. 2012. A communication theory of culture. In Anastacia Kurylo (ed.), Inter/ Cultural communication: Representation and construction of culture, 69-87. Thousand Oaks, CA: Sage.

Carbaugh, Donal, Sunny Lie, Liene Locmele, \& Nadezhda Sotirova. 2012. Ethnographic studies of intergroup communication. In Howard Giles (ed.), International communication association's handbook of intergroup communication, 44-57. New York \& Abingdon: Routledge.

Culpeper, Jonathan \& Michael Haugh. 2014. Pragmatics and the English language. Basingstoke: Palgrave Macmillan.

Derlega, Valerian, Sandra Metts, Sandra Petronio \& Stephen Margulis. 1993. Self-Disclosure. Thousand Oaks, CA: Sage.

Dindia, Kathryn. 2000. Self-disclosure, identity, and relationship development: A dialectical perspective. In Kathryn Dindia \& Steve Duck (eds.), Communication and personal relationships, 147-162. Chichester: Wiley.

Fuchs, Stephan. 2001. Against essentialism: A theory of culture and society. Harvard, MA: Harvard University Press.

Garfinkel, Harold. 1967. Studies in ethnomethodology. Englewood Cliffs, NJ: Prentice-Hall. Glenn, Philip. 2003. Laughter in interaction. Cambridge: Cambridge University Press.

Goddard, Cliff. 2006. “Lift your game Martina!": Deadpan jocular irony and the ethnopragmatics of Australian English. In Cliff Goddard (ed.), Ethnopragmatics: Understanding discourse in cultural context, 65-97. Berlin: Mouton de Gruyter.

Goddard, Cliff. 2009. Not taking yourself too seriously in Australian English: Semantic explications, cultural scripts, corpus evidence. Intercultural Pragmatics 6(1). 29-53.

Goddard, Cliff. 2012a. 'Early interactions' in Australian English, American English, and English English: Cultural differences and cultural scripts. Journal of Pragmatics 44(9). 1038-1050.

Goddard, Cliff. 2012b. Cultural scripts and communication style differences in three Anglo Englishes. In Barbara Kyrk-Kastovsky (ed.), Intercultural miscommunication: Past and present, 101-120. Amsterdam, the Netherlands: John Benjamins.

Haugh, Michael. 2010. Jocular mockery, (dis)affiliation, and face. Journal of Pragmatics 42(8). 2106-2119.

Haugh, Michael. 2011. Humour, face and im/politeness in getting acquainted. In Bethan Davies, Michael Haugh \& Andrew Merrison (eds.), Situated politeness, 165-184. London: Continuum.

Haugh, Michael. 2012. Conversational interaction. In Keith Allan \& Kasia Jaszczolt (eds.), The Cambridge handbook of pragmatics, 251-274. Cambridge: Cambridge University Press.

Haugh, Michael. 2014. Jocular mockery as interactional practice in everyday Anglo-Australian conversation. Australian Journal of Linguistics 34(1). 76-99.

Haugh, Michael. 2015. Im/politeness implicatures. Berlin: Mouton de Gruyter.

Hepburn, Alex \& Galina B. Bolden. 2013. The conversation analytic approach to transcription. In Tanya Stivers \& Jack Sidnell (eds.), The handbook of conversation analysis, 57-76. Oxford: Blackwell.

Holliday, Adrian. 1999. Small cultures. Applied Linguistics 20(2). 237-264.

Holt, Elizabeth. 2013. 'There's many a true word said in jest': Seriousness and nonseriousness in interaction. In Phillip Glenn \& Elizabeth Holt (eds.), Studies of laughter in interaction, 69-89. London: Bloomsbury. 
Jefferson, Gail. 1979. A technique for inviting laughter and its subsequent acceptance-declination. In George Psthas (ed.), Everyday language: Studies in ethnomethodology, 79-95. New York: Irvington.

Jefferson, Gail. 1984. On stepwise transition from talk about a trouble to inappropriately next-positioned matters. In J. Maxwell Atkinson \& John C. Heritage (eds.), Structures of social action, 191-222. Cambridge: Cambridge University Press.

Jefferson, Gail. 2004. Glossary of transcript symbols with an introduction. In Gene Lerner (ed.), Conversation analysis: Studies from the first generation, 13-23. Amsterdam, the Netherlands: John Benjamins.

Maynard, Douglas \& Don Zimmermann. 1984. Topical talk, ritual and the social organization of relationships. Social Psychology Quarterly 47. 301-316.

Pearce, W. Barnett \& Stewart M. Sharp. 1973. Self-disclosing communication. The Journal of Communication 23(4). 409-425.

Petronio, Sandra. 2002. Boundaries of privacy: Dialectics of disclosure. Albany, NY: State University of New York Press.

Pillet-Shore, Danielle. 2010. Making way and making sense: Including newcomers in interaction. Social Psychology Quarterly 73(2). 152-175.

Pillet-Shore, Danielle. 2011. Doing introductions: The work involved in meeting someone new. Communication Monographs 78(1). 73-95.

Pillet-Shore, Danielle. 2012. Greeting: Displaying stance through prosodic recipient design. Research on Language and Social Interaction 45(4). 375-398.

Pomerantz, Anita. 1988. Offering a candidate answer: An information seeking strategy. Communication Monographs 55. 360-373.

Potter, Jonathan \& Alexa Hepburn. 2010. Putting aspiration into words: 'Laugh particles', managing descriptive trouble and modulating action. Journal of Pragmatics 42(6). 1543-1555.

Rayson, Paul. 2008. From key words to key semantic domains. International Journal of Corpus Linguistics 13(4). 519-549.

Schegloff, Emanuel. 2001. Getting serious: Joke »> serious 'no'. Journal of Pragmatics 33. 1947-1955.

Schegloff, Emanuel. 2007. Sequence organization in interaction. Cambridge: Cambridge University Press.

Schneider, Klaus P. 1987. Topic selection in phatic communication. Multilingua 6(3). 247-256.

Schneider, Klaus P. 1988. Small talk: Analysing phatic discourse. Marburg: Hitzeroth.

Schneider, Klaus P. 2008. Small talk in England, Ireland, and the USA. In Klaus P. Schneider \& Anne Barron (eds.), Variational pragmatics: A focus on regional varieties in pluricentric languages, 99-139. Amsterdam, the Netherlands: John Benjamins.

Schneider, Klaus P. 2010. Variational pragmatics. In Mirjam Fried, Jan-Ola Östman, \& Jef Verschueren (eds.), Variation and change: Pragmatic perspectives, 239-267. Amsterdam, the Netherlands: John Benjamins.

Schneider, Klaus P. 2012. Appropriate behaviour across varieties of English. Journal of Pragmatics 44(9). 1022-1037.

Schneider, Klaus P. \& Anne Barron (eds.). 2008. Variational pragmatics: A focus on regional varieties in pluricentric languages. Amsterdam, the Netherlands: John Benjamins.

Scollo, Michelle. 2011. Cultural approaches to discourse analysis: A theoretical and methodological conversation with special focus on Donal Carbaugh's cultural discourse theory. Journal of Multicultural Discourses 6(1). 1-32. 
Silverstein, Michael. 1984. On the pragmatic 'poetry' of prose: Parallelism, repetition, and cohesive structure in the time course of dyadic conversation. In Deborah Schiffrin (ed.), Meaning, form and use in context: Linguistic applications, 181-199. Washington, DC: Georgetown University Press.

Stewart, Edward C. \& Milton J. Bennett. 1991. American cultural patterns. A cross-cultural perspective. Revised edn. Yarmouth, MN: Intercultural Press.

Stivers, Tanya. 2013. Sequence organization. In Tanya Stivers and Jack Sidnell (eds.), The handbook of conversation analysis, 191-209. Malden, MA: Blackwell-Wiley.

Stokoe, Elizabeth. 2010. 'Have you been married, or . . . ?': Eliciting and accounting for relationship histories in speed-dating interaction. Research on Language and Social Interaction 43(3). 260-282.

Svennevig, Jan. 1999. Getting acquainted in conversation. Amsterdam, the Netherlands: John Benjamins.

Svennevig, Jan. 2014. Direct and indirect self-presentation in first conversations. Journal of Language and Social Psychology 33(3). 302-327.

Tottie, Gunnel. 2002. An introduction to American English. Oxford: Blackwell.

Usami, Mayumi. 2002. Discourse politeness in Japanese conversation. Tokyo: Hituzi Syobo. 